\title{
Status Report on Aeroelasticity in the Vehicle Development for X-57 Maxwell
}

\author{
Jennifer Heeg, ${ }^{*}$ Bret K. Stanford,${ }^{\dagger}$ Carol D. Wieseman, ${ }^{*}$ Steven J. Massey, ${ }^{\dagger}$ James Moore, ${ }^{\ddagger}$ \\ NASA Langley Research Center \\ Hampton, VA 23681, USA \\ Roger Truax, ${ }^{\S}$ and Kia Miller ${ }^{\mathbb{I I}}$ \\ NASA Armstrong Flight Research Center \\ Edwards, CA 93523, USA
}

\begin{abstract}
Risk reduction is the objective of the X-57 Maxwell aeroelasticity team. The X-57, NASA's experimental electric propulsion aircraft, has a long thin wing with primary propulsion systems located at the wing tips and high lift motors distributed along the span. Many of the classical aeroelastic concerns associated with such a configuration were addressed through early design decisions. The as-designed intermediate flight vehicle configurations show flutter mechanisms associated with flexible models of control surface systems -the stabilator, flaps and ailerons. Improvements to the analytical models, based on ground test data and project decisions about flight operations, show improved prospects of the vehicle being aeroelastically stable throughout the flight envelope. On-going ground testing and further analyses will lend credibility to the flutter predictions and vehicle safety.
\end{abstract}

\section{Introduction}

NASA's Scalable Convergent Electric Propulsion Technology and Operations Research (SCEPTOR) project is developing a flight vehicle, the X-57 Maxwell, to demonstrate the increased efficiency associated with distributed electric propulsion systems. Distributed electric propulsion uses multiple electric motors to yield beneficial aerodynamicpropulsion interaction. In the SCEPTOR project, the flight vehicle will feature two types of this technology: large wing tip propellers that will operate at the cruise condition and interact with the wing tip vortex; and small high lift propellers along the wing leading edge to accelerate the flow for improved low speed performance.

Electric motors promise significant increases in vehicle efficiency as compared to internal combustion engines [1], but realizing those benefits depends on a unique wing structural design with complicated load paths and associated design challenges [2]. While the concept of distributed electric propulsion allows for a much smaller wing, many practical issues associated with structural design must be addressed. Among these issues are aeroelastic concerns.

Development of the X-57 has involved aeroelastic considerations from the project outset due to the configuration requirements of large wing tip propulsors and multiple leading edge high-lift propulsion systems conflicting with the requirement to make the wing thin and light. The challenge to the structures and aeroelasticity team is to balance the requirements and arrive at a structural design, which has sufficient safety margins but doesn't compromise the performance research objective. For the SCEPTOR project, we have taken the approach of modifying an existing aircraft, (the Tecnam P2006T) [3], reducing both cost and risk. The program has four configurations, each configuration advancing the complexity of the vehicle modification ("Mod"). Mod I was the baseline vehicle, without modification, used to establish baseline performance quantities. Mod I flight testing was completed in 2017; Mod II flight testing is scheduled for the early part of 2019. The Mod II configuration, shown in Figure 1a, has the propulsion system of the Tecnam replaced with the electric cruise motors. The replacement motors will be located at the same locations as the stock Tecnam propulsion system, that is, at inboard locations. Testing this configuration allows us to install and evaluate critical systems: the electric motors, battery systems and instrumentation. Mod II will establish flight safety aspects of the electric power systems and also provide performance data for the electric retrofit aircraft. The Mod III

\footnotetext{
* Research Engineer, Aeroelasticity Branch, AIAA Associate Fellow.

${ }^{\dagger}$ Research Engineer, Aeroelasticity Branch, AIAA Senior Member.

$\doteqdot$ Research Engineer, Computational Structures Branch, Member AIAA.

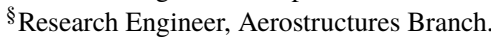

II Aerospace Engineer, Aerostructures Branch, Member AIAA.
} 


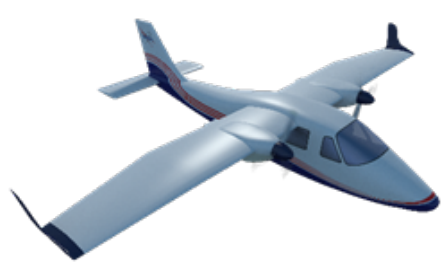

(a) Mod II

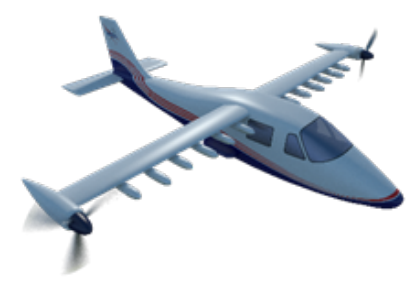

(b) Mod III

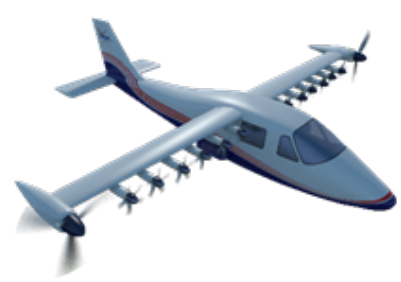

(c) Mod IV

Fig. 1 Vehicle configurations of the $\mathrm{X}-57$ project.

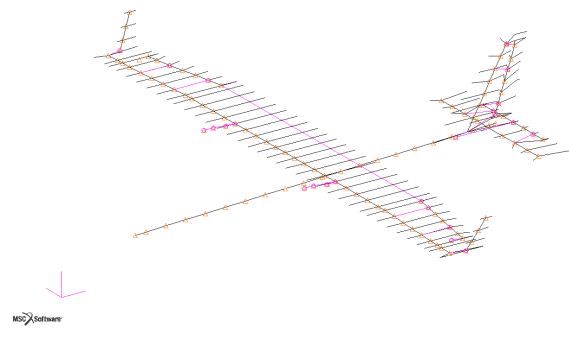

(a) Mod II

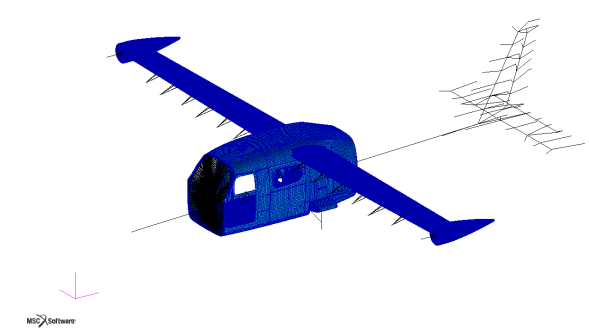

(b) Mod III

Fig. 2 Finite element models.

configuration, Figure 1b, will feature a newly designed and constructed wing, with the wing tip cruise motors. Mod III testing will achieve the primary research objective, demonstrating improved cruise efficiency. The high-lift motors will not be present in the Mod III configuration, replaced by dummy nacelles. The Mod IV configuration, Figure 1c, will have the high-lift motors and propellers. This configuration will address secondary objectives associated with acoustics, controls and certification.

At this point in time, June 2018, the project is performing ground testing of components for the Mod II and Mod III configurations and preparing to flight test the Mod II configuration. Hardware components for the Mod III wing are being fabricated. The aeroelastic tasks have migrated from weighing in on the Mod III wing design to evaluating the designed vehicle configurations, performing and assessing information from ground tests, refining analyses and models, and evaluating vehicle issues. This paper presents information in each of these areas. This paper will not cover the current status of whirl flutter work. Whirl flutter, considered a critical aeroelastic issue for these configurations, is not part of the present report. Current whirl flutter analysis results are presented in a companion paper by Hoover and Shen. [4].

\section{Aeroelastic evaluation of the vehicle configurations}

\section{A. Mod II}

Flutter analyses for the Mod II configuration have been performed using a classical beam model structural representation. The FEM, depicted in Figure 2a, has approximately 300 grid points and 600 elements, as summarized in Table 1. The FEM and the aerodynamic paneling used in the Mod II analyses are derivatives of models obtained from the retrofit aircraft manufacturer, Tecnam, based on the Tecnam P2006 prototype aircraft. Modifications to the Tecnam models were made to reflect the properties of the fleet aircraft rather than the prototype and to utilize preferred modeling practices. These modifications include changing from a beam spline to an infinite plate spline, modifying the aerodynamic planform box aspect ratios and eliminating gaps in the aerodynamic paneling at control surface hinge lines. The linear panel model, shown in Figure 3, does not include aerodynamic representations of the motor nacelles.

Additional changes were made to represent the Mod II hardware, particularly the conversion from the piston engines to the electric motors and the associated mounting hardware. Also, masses associated with the batteries were 
Table 1 Finite element models.

\begin{tabular}{clcc} 
Configuration & Description of FEM & Number of grid points & Number of elements \\
\hline \hline Mod II & Classical Beam Model & $\sim 300$ & $\sim 600$ \\
\hline Mod III & $\begin{array}{l}\text { Detailed wing and center fuselage models; } \\
\text { beam models for empennage, forward and } \\
\text { aft fuselage segments }\end{array}$ & $\sim 600,000$ & $\sim 14,000$ \\
\end{tabular}

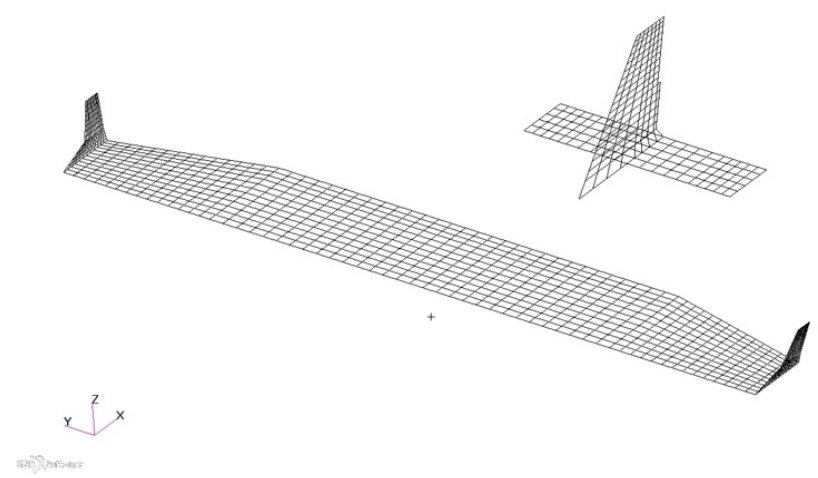

Fig. 3 Aerodynamic paneling of the Mod II configuration.

incorporated, while the masses associated with fuel, passengers and luggage were removed.

Assumed rigid body mode shapes at $0 \mathrm{~Hz}$ were used, and the pilot control stick was modeled as rigidly fixed. The in-vacuo structural dynamic modal frequencies and mode shapes are listed in Table 2. There were two structural dynamic modes with substantial stabilator rotation in the original FEM: one at $13.0 \mathrm{~Hz}$ and a second mode at $15.59 \mathrm{~Hz}$, where there was considerable participation of the fuselage first bending mode.

Based on a preliminary ground vibration test ("mini-GVT") that will be discussed later in this paper, the stabilator stiffness was increased by approximately $70 \%$, increasing the stabilator rotational frequency by approximately $30 \%$, to $17.0 \mathrm{~Hz}$, above the combined stabilator-fuselage modal frequency. The frequency of the combined stabilator-fuselage modal frequency dropped to $15.2 \mathrm{~Hz}$.

Flutter analyses were performed using ZAERO [5] at matched point conditions at Mach 0.3, using 25 structural modes (up to $63 \mathrm{~Hz}$ ) and assuming zero structural damping. Flutter analysis results from before and after the GVT-based update of the stabilator stiffness are shown in Figure 4. The upper plot shows the damping versus equivalent airspeed and the bottom plot shows the migration of the aeroelastic modal frequencies. The before-update results are shown in grey. The after-update results are shown by the colored lines. Three modes are highlighted by thicker lines: symmetric wing first bending (red); stabilator rotation (orange) and stabilator rotation + fuselage vertical first bending (purple). Two vertical blue lines show important velocities; the dashed line shows the dive speed and the solid line shows the required analysis clearance condition, which is $20 \%$ above the dive speed.

The stability boundary, the line of zero damping, is shown on the upper plot. Positive values of damping are unstable. The results show a hump mode (tendency toward instability over a finite range of velocities followed by a tendency towards stability) associated with modes that contain stabilator rotation. The aeroelastic hump mode is shown to be nearly neutrally damped, both before and after updating the stabilator stiffness. The before-update case indicates a mild instability for airspeeds between 100 and 270 kts.

This hump mode was viewed as a mode to monitor, but not necessarily as a real instability that would prevent flight testing. The analysis was performed assuming that the structure contributes no damping. Assumptions of modal structural damping of 1-3\% are a common practice. The observed hump mode is stabilized by assuming approximately $2 \%$ structural damping $\left(\zeta_{\text {strucural }}=0.01\right)$. In addition to the low damping characteristic, the analysis results indicate that the onset of the instability is gentle with regard to increasing airspeed. If the instability is an issue for the flight vehicle, careful envelope clearance procedures of a well-instrumented and monitored vehicle should indicate the incipient problem and allow the test team to back away from the instability. The presence of this hump mode and the large 
Table 2 Mode shapes and frequencies from the Mod II FEM.

Finite element model, InMode shape

Symmetric (S) or Anti-symmetric (A) vacuo modal natural frequencies, $\mathbf{H z}$

\begin{tabular}{cll}
\hline \hline \multicolumn{1}{c}{ 6 Rigid Body Modes } & \\
\hline 7.93 & Wing 1st Vertical Bending & $\mathrm{S}$ \\
\hline 10.15 & $\begin{array}{l}\text { Fuselage 1st Lateral Bending + Aft Fuselage Tor- } \\
\text { sion }\end{array}$ & $\mathrm{A}$ \\
\hline 12.95 & Wing 1st Vertical Bending + Aft Fuselage Torsion & $\mathrm{A}$ \\
\hline $13.00(17.0)$ & Stabilator Rotation (GVT-updated value) & $\mathrm{S}$ \\
\hline $15.59(15.2)$ & $\begin{array}{l}\text { Stabilator Rotation + Fuselage 1st Vertical Bending } \\
- \text { Out of Phase (GVT-updated value) }\end{array}$ & $\mathrm{S}$ \\
\hline 16.06 & $\begin{array}{l}\text { Aft Fuselage Torsion + Wing 2nd Vertical Bending- } \\
\text { In Phase }\end{array}$ & $\mathrm{A}$ \\
\hline 19.63 & $\begin{array}{l}\text { Vertical Tail 1st Lateral Bending + Aft Fuselage } \\
\text { Torsion }\end{array}$ & $\mathrm{A}$ \\
\hline 19.92 & Rudder Rotation & $\mathrm{A}$ \\
\hline 23.99 & $\begin{array}{l}\text { Wing 2nd Vertical Bending + Fuselage 1st Vertical } \\
\text { Bending + Stabilator 1st Vertical Bending }\end{array}$ & $\mathrm{S}$ \\
\hline 25.57 & Aileron Rotation & A \\
\hline 29.16 & $\begin{array}{l}\text { Stabilator 1st Vertical Bending with Rotation }+ \\
\text { Fuselage 2nd Vertical Bending- Out of Phase }\end{array}$ & A \\
\hline 33.31 & $\begin{array}{l}\text { Stabilator 1st Vertical Bending with Rotation + } \\
\text { Fuselage 2nd Vertical Bending + Wing 1st in-plane } \\
\text { Bending }\end{array}$ & $\mathrm{S}$ \\
\hline
\end{tabular}




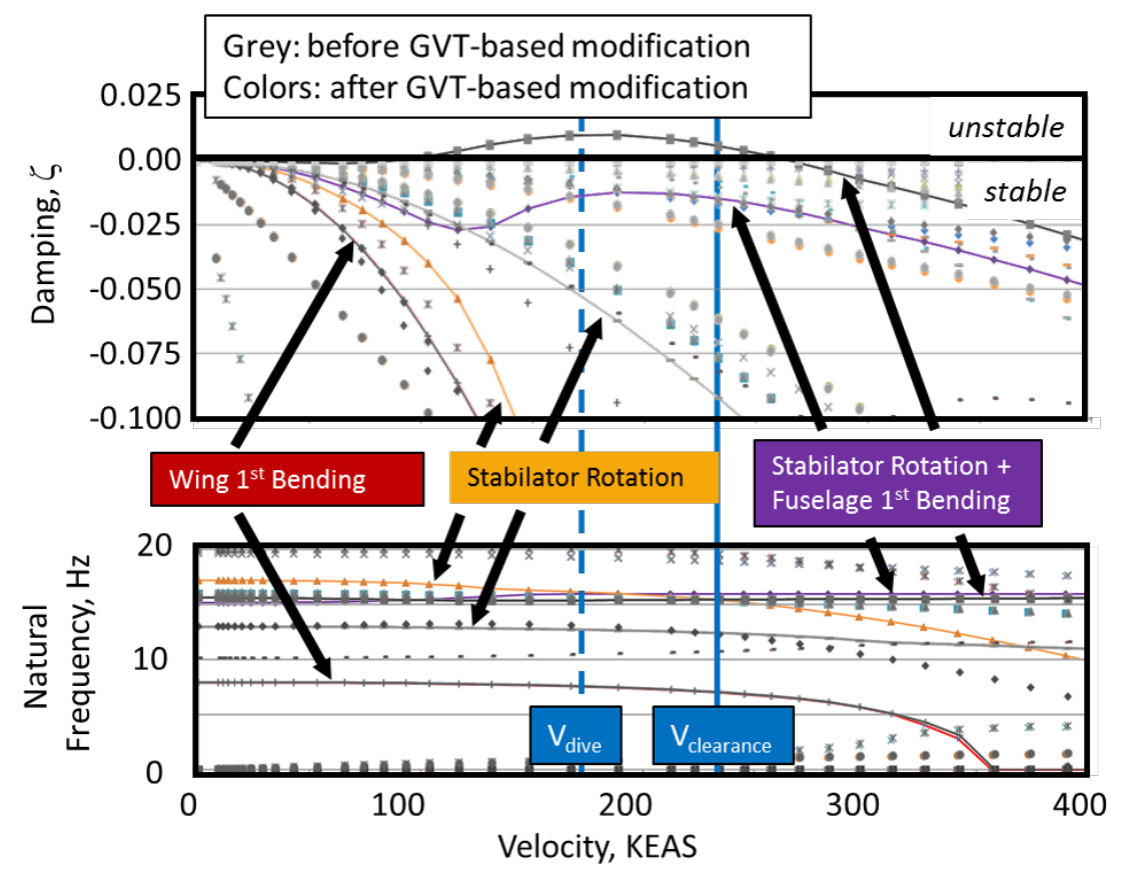

Fig. 4 Flutter analysis results, Mod II.

number of modes with aft fuselage participation led the team to request a preliminary mini-GVT of the incomplete Mod II vehicle. The dataset from the mini-GVT was used to provide an update to the stabilator rotational stiffness. The after-update results, colored lines in Figure 4, show the existence of the hump mode, but in this case, there is sufficient aerodynamic damping such that the mode does not destabilize. Note that the hump mode is not the pure stabilator rotation, but rather a coupling between empennage motion and fuselage bending.

In both analyses, the stabilator frequency drops as the flight speed increases, as shown in the lower plot of Figure 4. Interestingly, the after-update analysis shows that the hump mode is stabilized, even though the frequencies of the stabilator rotation and the combined stabilator and fuselage modes cross. There is a small inflection in each modal frequency curve at the onset of the hump mode instability, indicating that there is some modal coupling. With increasing airspeed, the coupling diminishes and the hump mode becomes more stable.

Mod II flutter analyses indicate that the vehicle will be flutter-free throughout the flight envelope and beyond the analysis clearance requirements (20\% above the dive velocity). However, the aeroelastic analyses performed to date indicate that critical parameters include stabilator rotational stiffness, the fuselage weight and center of gravity. These parameters will be treated non-deterministically, using input uncertainty propagation, to evaluate the flutter margins of the flight vehicle. During ground vibration testing comparisons, attention will be paid to the new systems of the aircraft (the motors and mount systems), the changes due to relocation of heavy components (the batteries), the stabilator and the aft portion of the vehicle.

\section{B. Mod III}

The flutter assessment process for the Mod III configuration differs from the Mod II flutter assessment process, although both rely heavily on proven linear analysis tools. During the Mod III wing design process, it was required to have quick turn-around on flutter predictions for each design iteration. To speed this process, a tool chain was established to start with an aerodynamic shape (outer mold line or OML) and structural layout. With this tool suite, the wing design was rapidly converted from a CAD design into a structural finite element model and then converted to the linear aeroelastic analysis components. [2] This tool chain has been enabling to the SCEPTOR project. 


\section{Finite element structural model}

As the vehicle components have gone through detailed design, these systems have been incorporated into the structural and aerodynamic models. The current FEM is the first model iteration that incorporates the flap systems, aileron systems, aft fuselage and empennage. One of the inherent characteristics of the tool chain discussed above is that a highly complex structural model is used as the basis for aeroelastic analysis, rather than a simplified structural dynamics model. The difference in the complexity between the Mod II and the Mod III finite element models can be observed in Figure 2b. While the Mod II configuration uses a classical beam model, the Mod III model incorporates detailed structures of the wing, tip nacelle, control surfaces and systems and central fuselage. The aft fuselage and empennage modeling is taken directly from the Mod II finite element model. The Mod III FEM contains significantly more grid points and elements than the Mod II FEM, as detailed in Table 1, which adds the additional burden of significantly longer computational times.

A more traditional simplified structural dynamic model allows for "clean" modes to be identified and their influence on flutter mechanisms readily observed. In using a complex structural model, modes often contain various components of vehicle motion, some of which do not contribute to external shape and thus to the fluid structure coupling. In a classical structural dynamic model, these modes are usually eliminated. Their presence in the Mod III FEM complicates interpretation of the physics at play in the flutter mechanisms and increases computational time. The Mod III FEM, as provided to the aeroelasticity team, had some modeling and design aspects that were investigated. Flutter analyses will be presented in this paper for the original and revised finite element models. All of the Mod III FEMs used in the present linear analyses are full-span, free-free models. Thus, the first six modes are rigid body modes.

\section{Linear aerodynamic model}

The aerodynamic panel mesh used for steady and unsteady doublet lattice computations is shown in Figure 5, and consists of 4,700 boxes across the wing, high-lift pods, cruise pods, vertical tail, and horizontal tail. The pods are approximately modeled as flat plates, in-line with the wing, when in reality they are non-lifting surfaces located (in the case of the high-lift pods) below the wing, connected with pylon structures. Infinite plate splines were used to communicate the information between the structure and the aerodynamic panels. The Mod III splining on the wing employs only the "hard points" of the structure, that is, nodes that lie at the intersection of ribs and skins or spars and skins. The splining on the nacelles employs fictitious structure comprised of rigid body elements (RBEs).

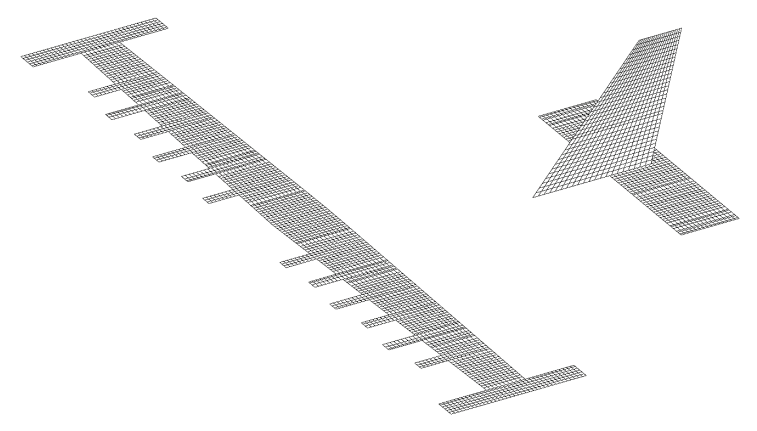

Fig. 5 Flat plate aerodynamic panel mesh for doublet lattice computations. 


\section{Linear flutter analysis of the Mod III original model}

Flutter analyses were conducted using MSC NASTRAN ${ }^{\mathrm{TM}}$ [6] and postprocessed using scripting languages, FORTRAN and MATLAB ${ }^{\mathrm{TM}}$ [7]. The Mod III nominal cruise Mach number, 0.233, was used and zero structural damping was assumed. The results, shown in Figure 6, are presented in a manner similar to the Mod II results. The upper plot is the damping versus equivalent airspeed. The stability boundary is again shown by the line of zero damping. Each of the grey and colored lines shows the damping of an aeroelastic mode as the airspeed increases. The thicker colored lines show seven particular modes that are discussed in more detail. The legend specifies the aeroelastic mode number and the frequency of the structural dynamic mode where that aeroelastic mode originated. Note that modes shown are aeroelastic modes, which are linear combinations of the structural dynamic modes. Modal participation factors for each mode are not presented in the current paper, but they are used to determine the modal content of each of the aeroelastic modes and flutter mechanisms.

The flutter results are also presented as a root locus plot in Figure 7; this plot is a different way of presenting the same information that appears in Figure 6. The eigenvalues, complex numbers, are plotted as real parts versus imaginary parts. Each aeroelastic eigenmode starts at the air-off eigenvalue obtained from a normal modes analysis (or structural dynamic eigensolution). The flutter root locus shows the migration of the eigenvalues of the aeroelastic modes as dynamic pressure or equivalent airspeed is increased. The vertical axis (where the real part of any eigenvalue is zero) is the boundary between stable and unstable behavior, with the right half plane being unstable.

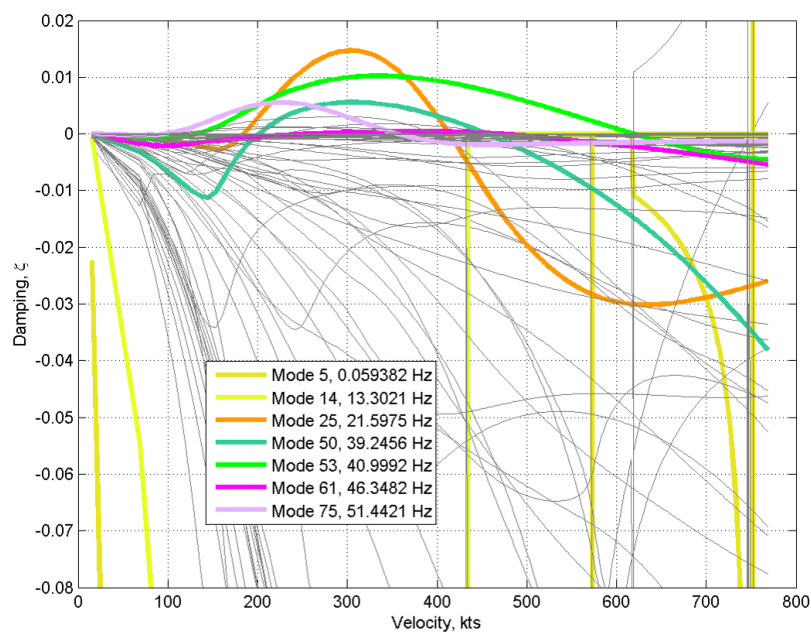

(a) Damping vs Equivalent airspeed

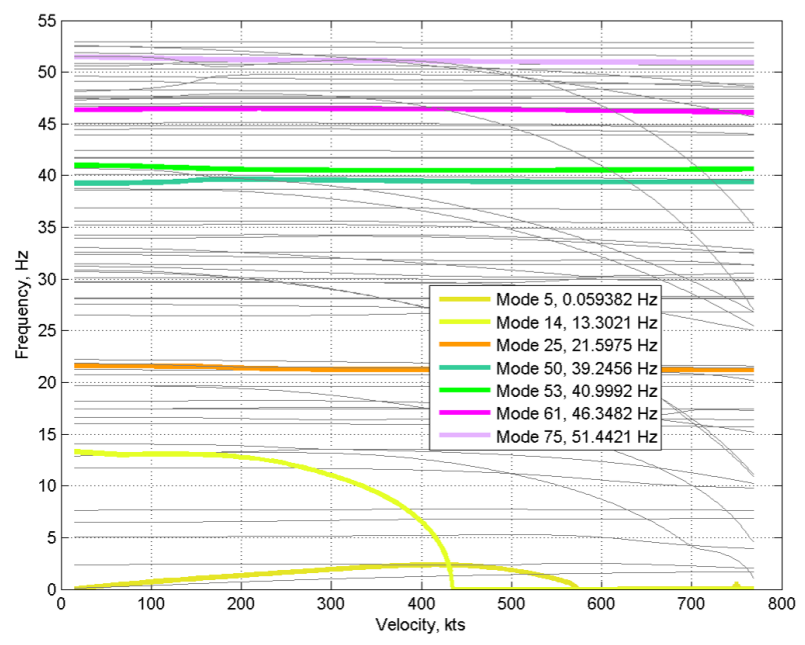

(b) Frequency vs Equivalant airspeed

Fig. 6 Flutter results for Mod III, original model.

The required analysis clearance equivalent airspeed for the Mod III vehicle is 200 KEAS. Within the clearance envelope, the original Mod III model has nine unstable flutter mechanisms. Five of the mechanisms are associated with the ailerons. Example unstable aileron modes are highlighted in the flutter eigenvalue plot (Modes 61 and 75 in Figures 6 and 7) and the flutter mode shape for aeroelastic mode 75 is shown in Figure 8a. Two flutter mechanisms are produced by flap modes (Modes 50 and 53 in Figures 6 and 7; flutter mode shape for mode 50 is shown in Figure 8b). One flutter mechanism is comprised of combined empennage motion and aft fuselage motion (Mode 25 in Figures 6 and 7 and Figure 8c). The ninth instability observed within the clearance envelope is the stabilator rotation (Mode 14 in Figures 6 and 7, which is a very low damping hump mode, similar to that observed in the Mod II flutter analysis prior to the update to the stabilator stiffness. Wing divergence (Mode 5 in Figures 6 and 7) and stabilator divergence (Mode 14 in Figures 6 and 7) occur well outside the flight envelope at 570 and 430 KEAS, respectively.

The most unstable mode (highest positive damping) is aeroelastic mode 25, aft fuselage torsion and empennage deformation. This is consistent with the Mod II flutter mechanism observed. The modes that go unstable at the lowest equivalent airspeeds (or dynamic pressures) are aileron modes. The lowest one, aeroelastic mode 75, destabilizes at 69 KEAS.

In the following sections, modeling of the aileron and flap systems are examined and modified. The stabilator stiffness is also updated identically to the update performed for the Mod II configuration, increasing the stiffness by $70 \%$. 


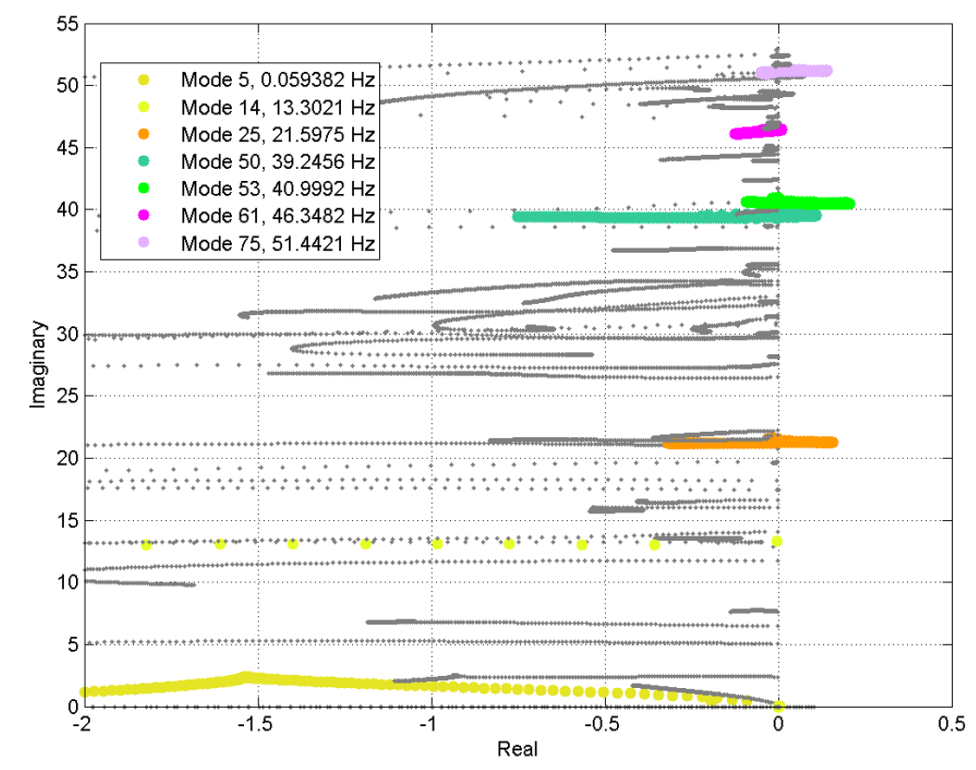

Fig. 7 Flutter root locus for Mod III, original model.

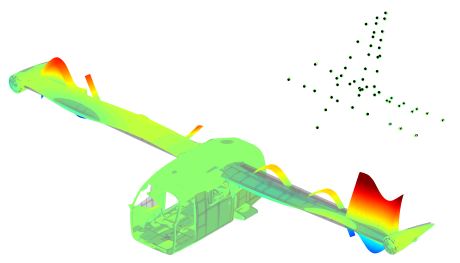

(a) Aeroelastic Mode 75, Aileron flutter

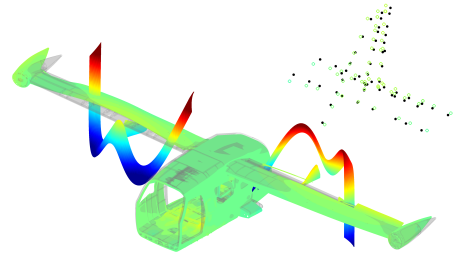

(b) Aeroelastic Mode 50, Flap flutter

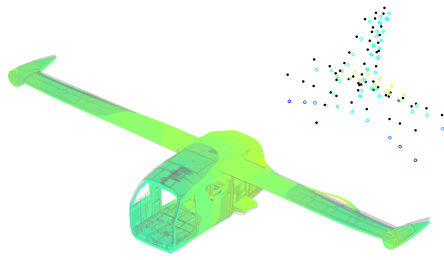

(c) Aeroelastic Mode 25, Empennage-Aft fuselage flutter

Fig. 8 Flutter mode shape examples for Mod III, original model.

\section{Aileron mass balancing}

For this class of vehicle, the mass balancing of the ailerons is often adjusted in the field prior to first flight, accounting for small mass changes such as paint or instrumentation. Small inaccuracies in the mass balance can produce aileron instabilities. The original version of the structural model had a small offset in the aileron/balance mass center of gravity, producing numerous flutter mechanisms. An aileron mass balancing study was performed to remove these modes in a manner similar to the process that will be used in the field to balance the aileron. Although very simple mass balancing works for most aircraft of this class, the long thin wing makes it possible that mass balancing could create unintended modal couplings.

Each aileron has eight concentrated masses acting to move the center of gravity (cg) of the combined aileron/actuator/balance mass system to lie near the aileron hinge line. The cg position in the chord-wise direction is calculated by Equation 1. The aileron balance percentage is calculated using Equation 2. The aileron is exactly, $100 \%$, balanced when the cg and hinge line coincide. Table 3 shows the parameters that appear in the equations, as well as providing the values used in the original FEM and the values chosen after completing aileron mass balance studies.

$$
\begin{gathered}
x_{c g}=\frac{M_{c s} * x_{c g, c s}+M F+M_{C O N M} *\left(x_{c g, C O N M}+X 1\right)}{M_{c s}+M F * M_{C O N M}} \\
A B=100 *\left(1-\frac{x_{c g}-x_{H L}}{c_{r e f}}\right)
\end{gathered}
$$


Table 3 Aileron mass balancing parameters.

\begin{tabular}{llll} 
Symbol Units & $\begin{array}{l}\text { Original quanti- } \\
\text { ties }\end{array}$ & $\begin{array}{l}\text { Modified quanti- } \\
\text { ties }\end{array}$ \\
\hline
\end{tabular}

\begin{tabular}{|c|c|c|c|c|}
\hline $\begin{array}{l}\text { Mass of Control sur- } \\
\text { face without mass bal- } \\
\text { ance masses }\end{array}$ & $m_{c s}$ & slinches $\left(l b_{f}-s^{2} /\right.$ inch $)$ & 0.004289 & 0.004289 \\
\hline CG of the Control surface & $x_{c g, c s}$ & inches & 168.81 & 168.81 \\
\hline $\begin{array}{l}\text { Total Mass of concen- } \\
\text { trated balance masses for } \\
\text { each aileron }\end{array}$ & $M_{C O N M}$ & slinches & 0.00575 & 0.00978 \\
\hline $\begin{array}{l}\text { CG of the concentrated } \\
\text { balance masses }\end{array}$ & $x_{c g, C O N M}$ & inches & 165.97 & 165.97 \\
\hline $\begin{array}{l}\text { Individual concentrated } \\
\text { balance mass ( } 8 \text { per } \\
\text { aileron) }\end{array}$ & & slinches & 7.19E-04 & $1.22 \mathrm{E}-03$ \\
\hline $\begin{array}{l}\text { Weight of individual } \\
\text { concentrated balance } \\
\text { mass (mass } * 32.2 \mathrm{ft} / \mathrm{s}^{2} * \\
12 \mathrm{in} / \mathrm{ft})\end{array}$ & & lbf & 0.278 & 0.472 \\
\hline $\begin{array}{l}\text { Shift location of each con- } \\
\text { centrated balance mass }\end{array}$ & $\mathrm{X} 1$ & inches & 0 & 0 \\
\hline $\begin{array}{l}\text { Mass Factor applied to } \\
\text { concentrated balance } \\
\text { masses }\end{array}$ & MF & & 1 & 1.7 \\
\hline $\begin{array}{l}\text { Hinge line location in the } \\
\text { chordwise direction }\end{array}$ & $x_{H L}$ & inches & 166.857 & $\begin{array}{l}166.857 \text { (varies } \\
\text { from } 166.785 \text { to } \\
166.894 \text {, depend- } \\
\text { ing on span loca- } \\
\text { tion considered) }\end{array}$ \\
\hline Aileron mean local chord & $c_{\text {ref }}$ & inches & 6.43 & 6.43 \\
\hline Aileron balance & $\mathrm{AB}$ & $\%$ & 94 & 100.3 \\
\hline
\end{tabular}


Moving the combined system cg can be accomplished by either adding mass or increasing the moment arm (distance to) the balance masses. Both of these quantities were varied and changes in trends in stability examined. Example stability results are shown in Figure 9. This plot is a root locus plot, zoomed in to focus on the primary modes associated with aileron flutter. The black symbols show the behavior when the aileron is $100 \%$ mass balanced by increasing the amount of balance mass. The red symbols show the results for a slightly overbalanced aileron (100.3\%). This latter case was chosen as the modified baseline mass balancing to incorporate into the FEM.

The study showed that the aileron can be mass balanced to eliminate the aileron flutter issues observed in the original analyses. It is required that the control surface be balanced to between 99 and $101 \%$. When balancing the aileron in the analysis, it was important to include the mass of the actuator in calculating the mass properties. Neglecting the actuator mass results in a $6 \%$ error in the mass balance calculations, making the aileron highly unstable and resulting in a large number of low velocity aileron flutter mechanisms.

In balancing the aileron, adding mass has a slightly different effect than relocating the mass relative to the hingeline. Predictably, adding mass decreases the natural frequencies of wing torsional modes. There is also a secondary effect on the frequencies of the 3rd bending modes due to the proximity of the aileron to the outboard node line of these modes.

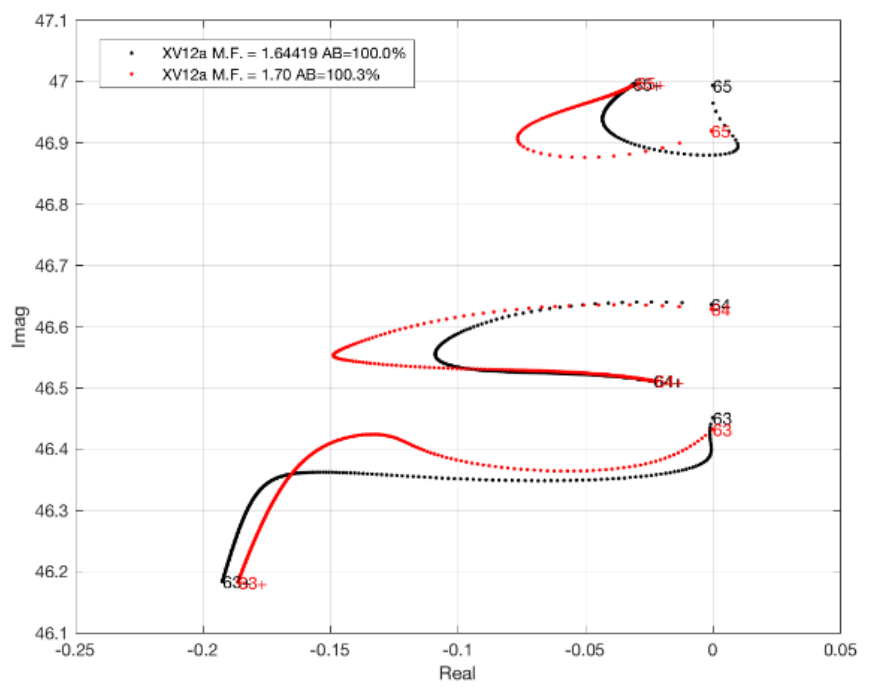

Fig. 9 Root locus of aileron mass balancing cases.

\section{Flap model modification for cruise configuration}

The flutter analysis discussed above showed two flutter mechanisms consisting of flap deformations. Although the original FEM included flaps that was in the stowed position, there were no restraining elements that might be expected to represent a stowed condition. In order to simulate the restraints for the stowed condition of the trailing edge flaps, two sets of rigid body connections are implemented. The first is shown in Figure 10, and connects each of the flap hinges ( 3 per flap) to the corresponding flap stop. This prevents rigid body motions, but the flap structure may still experience local bending deformation in between these connection points. Such deformation, during a stowed condition, is impossible for up-bending, as the flap is stowed under the wing cove. Negative deformation is still possible, though would be counteracted by the aerodynamic lift. In order to approximate this restraint, a line of rigid body connections (seen in Figure 11) is stitched along the wingspan, connecting the lower surface of the wing cove to the upper surface of the flap, completely removing any relative deformation between the two structures.

\section{Linear flutter results for the revised model}

Flutter results for the revised model are shown in Figure 12 and 13 . The blue vertical lines in Figure 12 represent velocities of interest: nominal cruise (dotted) and the analysis clearance requirement (solid). Flutter mechanisms associated with the aileron rotation have been nearly eliminated in the current analysis models by proper aileron mass balancing. A slightly unstable aileron hump mode (aeroelastic mode 53, shown in green in Figures 12 and 13 ) is still present over a range of low velocities. The maximum damping of this mode is 0.00069 , which indicates that this 


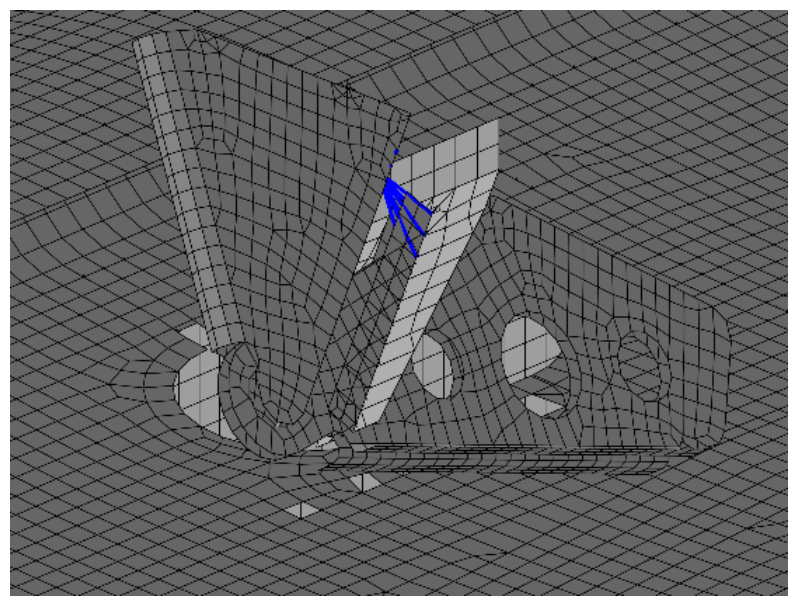

Fig. 10 Flap stop connections used to prevent rotation of the trailing edge flap mechanism.
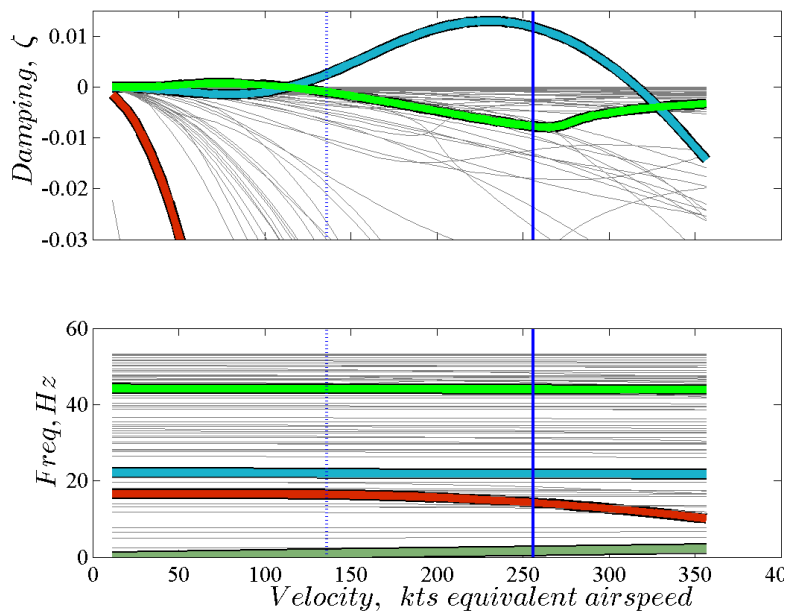

Fig. 12 V-g and V-f plots for Mod III, updated.

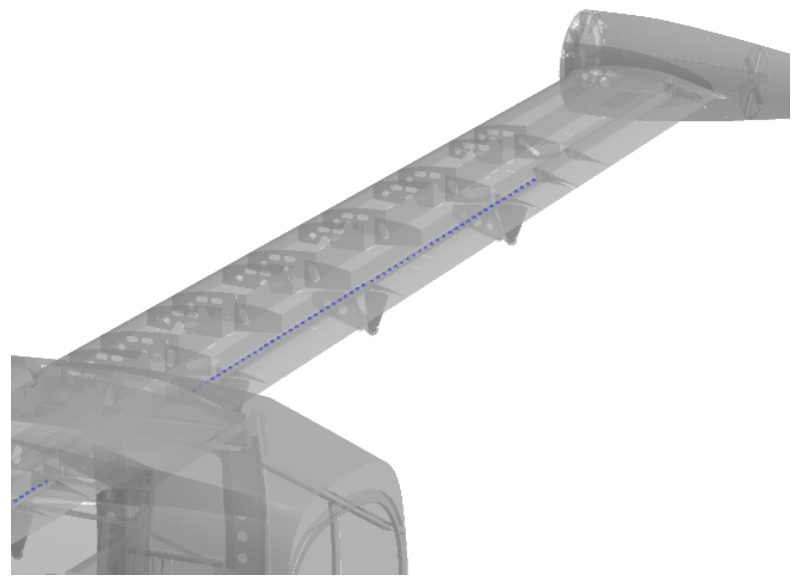

Fig. 11 Line of flap-wing connections used to prevent bending deformation of the trailing edge flap.

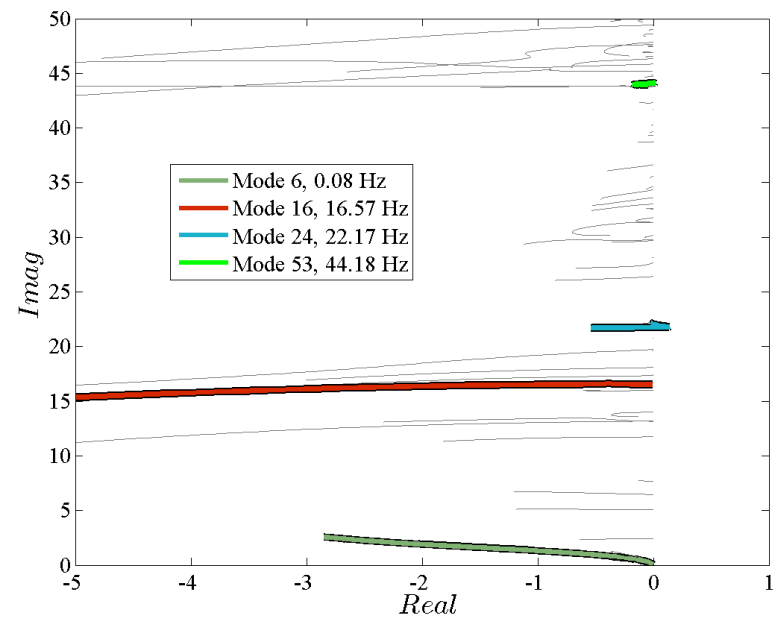

Fig. 13 Flutter root locus of Mod III, updated.

predicted instability will be overcome by structural damping. The flutter mechanisms associated with the flap system have been eliminated by the modeling constraints associated with stowing the flap. The remaining flutter mechanism has contributions from the aft portion of the fuselage and the stabilator rotational degree of freedom (aeroelastic mode 24, shown in blue in Figures 12 and 13). This is the same flutter mechanism present in the Mod II analysis results prior to updating the stabilator stiffness. For the Mod III configuration, however, this hump mode instability does not completely stabilize with the stiffness update.

\section{CFD}

For the Mod III configuration, the linear analysis has been augmented by unsteady Reynolds-averaged Navier-Stokes (URANS) simulations with fluid-structure coupling. These simulations are time-consuming and expensive, so they are conducted at only a few flight conditions. These higher fidelity simulations are used for evaluating the influence of physics not present in the linear modeling, benchmarking the linear results and providing confidence in assessments of the configuration being free of flutter. Rigid steady simulations are also performed to provide data to correct the linear analyses, including the influence of the rotating wing tip propellers. 


\section{CFD grid}

Building on the grid family reported in Massey et al. [8], a new grid was constructed starting from the medium grid (12.9M nodes), with more refinement in the pylon regions, shown in Figure 14. The grid was created using Pointwise $^{\mathrm{TM}}$ [9], following the recommended best practices established from the 6th AIAA Drag Prediction workshop [10]. Within the viscous layer, the elements were sized to maintain a $y^{+} \approx 1$. Tetrahedrons in the boundary layer were merged into triangular prisms to improve turbulence model performance. Farfield boundaries are located on a hemisphere 100 semispan lengths away from the wing surface. The resulting grid used in current work consists of $18 \mathrm{M}$ nodes.

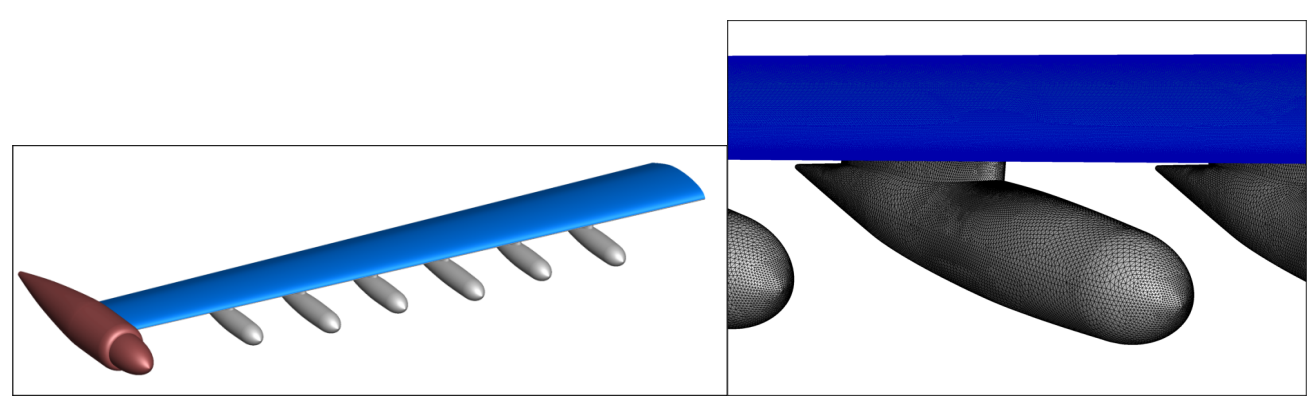

(a) Mesh based on prior medium mesh

(b) Nacelle and pylon regions have been refined

Fig. 14 CFD mesh used in current work.

\section{CFD solver}

Solutions to the Reynolds-averaged Navier-Stokes equations are computed using the FUN3D [11] flow solver. All solid surfaces are modeled with no-slip boundary conditions and farfield boundaries are of the Riemann invariant type. Turbulence closure is obtained using the Spalart-Allmaras [12] one-equation model with the quadratic constitutive relation Reynolds stress model of Spalart [13] and the Spalart-Shur rotation/curvature correction [14]. Inviscid fluxes are computed using the Roe scheme [15]. For second-order spatial accuracy, interface values required for Roe's scheme are reconstructed using gradients at grid nodes obtained by a least-squares technique. For the mixed prismatic/tetrahedral grids used in this study, the viscous fluxes are evaluated to second-order accuracy using a combination of edge-based and Green-Gauss gradients. In high gradient regions of the flow, limiters on these reconstructed values may be needed for stability. However, in the present computations, no limiters were required. Time integration is accomplished by an Euler implicit backwards difference scheme with dual time stepping to achieve second-order accuracy [16, 17]. Rigid solutions were generated using a nondimensional time step of 1 with 6 subiterations, corresponding to a physical time step of $7.68 \mathrm{E}-5 \mathrm{sec}$ or $13 \mathrm{kHz}$. The static aeroelastic solutions were performed increasing the number of subiterations to 10.

Grid motion due to aeroelastic deformations is carried out by treating the CFD grid as a linear elastic medium with material properties based on the grid characteristics with appropriate application of the Geometric Conservation Law [18]. In the present computations, the modulus of elasticity was boosted near the wing by setting it to vary with the inverse of the square of the distance away from the wing surface, thereby increasing the robustness of the grid motion.

The structural dynamics are computed internally using a linear modal method, which was implemented in FUN3D by Biedron and Thomas [19] following the approach used in CAP-TSD [20]. Because of the difference between the FEM and CFD surface definitions, a radial basis function interpolation scheme [21] was implemented to smoothly interpolate the FEM modes to the CFD grid. A total of 12 mode shapes computed using NASTRAN, were used in the FUN3D computations.

\section{3. $C F D$ results \& applications}

Flutter simulations using the modified Mod III structural dynamic modes are in progress. Steps in performing the dynamic aeroelastic calculations include calculating the rigid steady results and performing static aeroelastic simulations. The static aeroelastic simulations have been completed. The wing deformation at a dynamic pressure of $252 \mathrm{psf}$ is shown in Figure 15. At this condition, the tip deflection is approximately 8 inches in translation and $1.5^{\circ}$ in rotation.

CFD simulations have also been conducted to supply data for calculating correction factors for the linear aeroelastic 


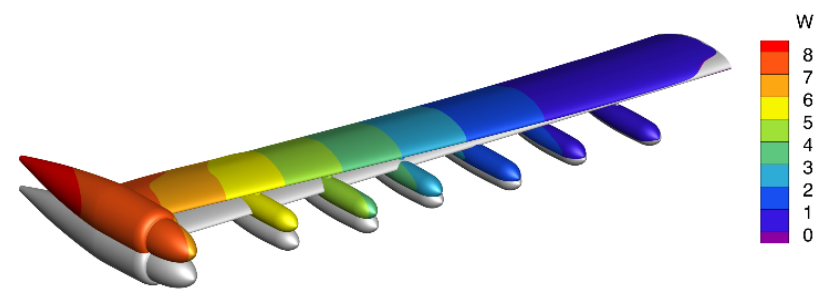

Fig. 15 Static aeroelastic displacement of Mod III wing at Mach 0.2706, dynamic prsesure 252 psf.

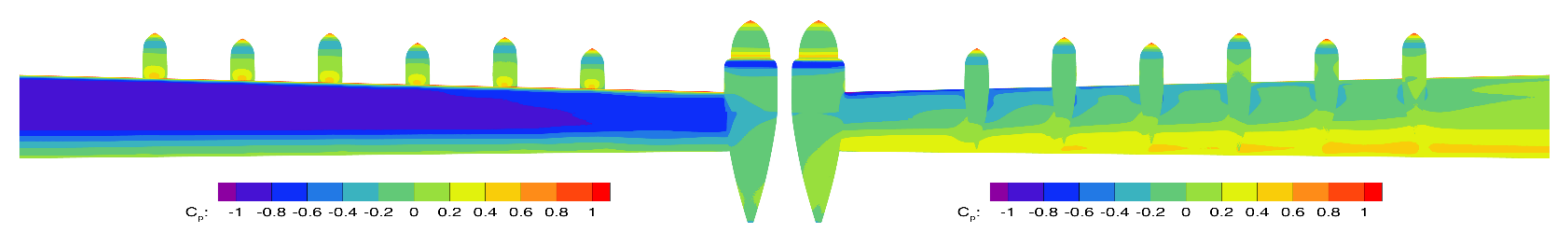

(a) Upper surface

(b) Lower surface

Fig. $16 C_{p}$ distributions from Navier-Stokes steady rigid analysis, Mach 0.2706.

analysis. Two sets of correction factors are desired: one to correct the unpowered configuration and one to correct the configuration to represent the propeller operating at the nominal cruise condition at $2250 \mathrm{RPM}$. Correction factors are based on steady rigid CFD solutions, exemplified by the pressure coefficients shown in Figure 16 for the unpowered configuration at Mach 0.2706 . To generate the correction factors, $\Delta C_{p}$ distributions have to be calculated using the upper and lower surface pressures from the CFD. The first approach taken to develop correction factors was a direct ratio of the pressures of CFD to the pressures from the linear aerodynamics. These results are shown in Figure 17. The direct ratio method resulted in very large correction factors (magnitudes as high as 100) near the wing leading edge and near the intersections of the wing and high lift pylons. The flutter analysis based on these correction factors showed classical bending-torsion instability at very near zero airspeed. The underlying cause of the instability is the large correction factors, which correspond to regions where the sign of the correction factors change, Figure 17d. Several improved methods for generating adequate correction factors are being explored and show promise.

As part of the correction factor generation, the CFD simulations with and without the propeller operating have been compared. For the powered cruise case, wing tip propellers were modeled using an actuator disk representation, with thrust and torque coefficient data used for inputs. The thrust and torque coefficient data were derived from an XROTOR [22] blade element momentum analyses. The difference between the pressure coefficient distribution is 


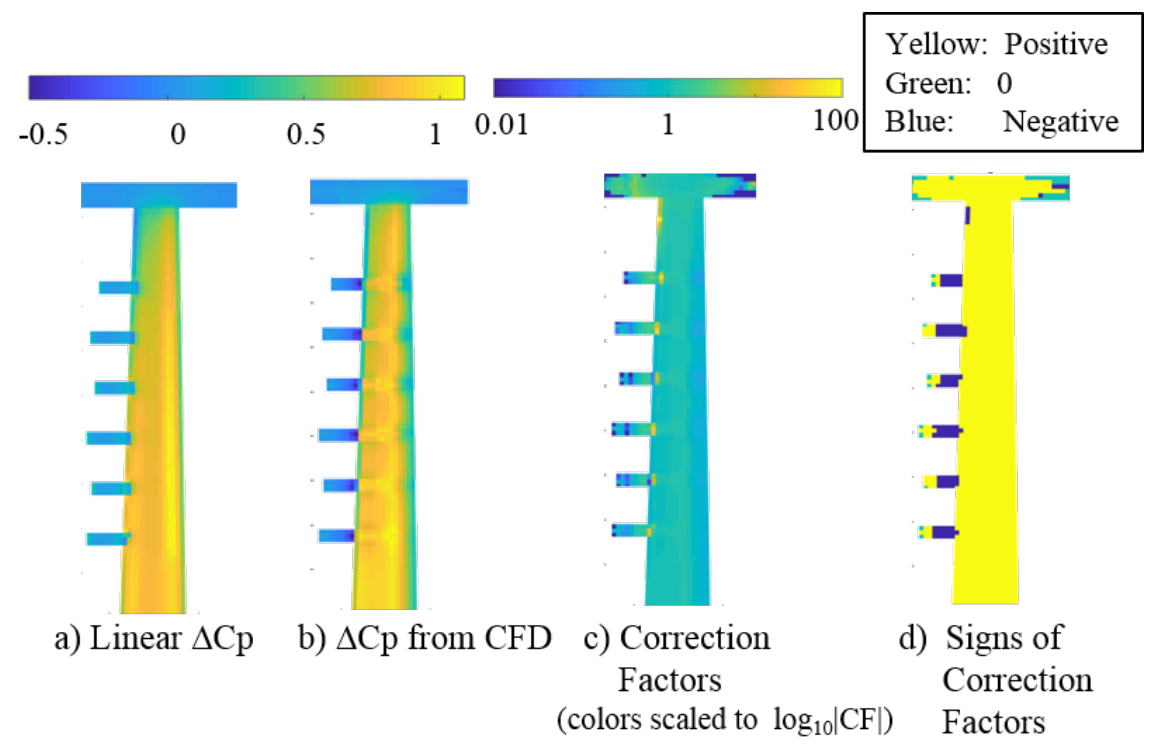

Fig. 17 Correction factors generated by direct ratios of $\Delta C_{p}$.

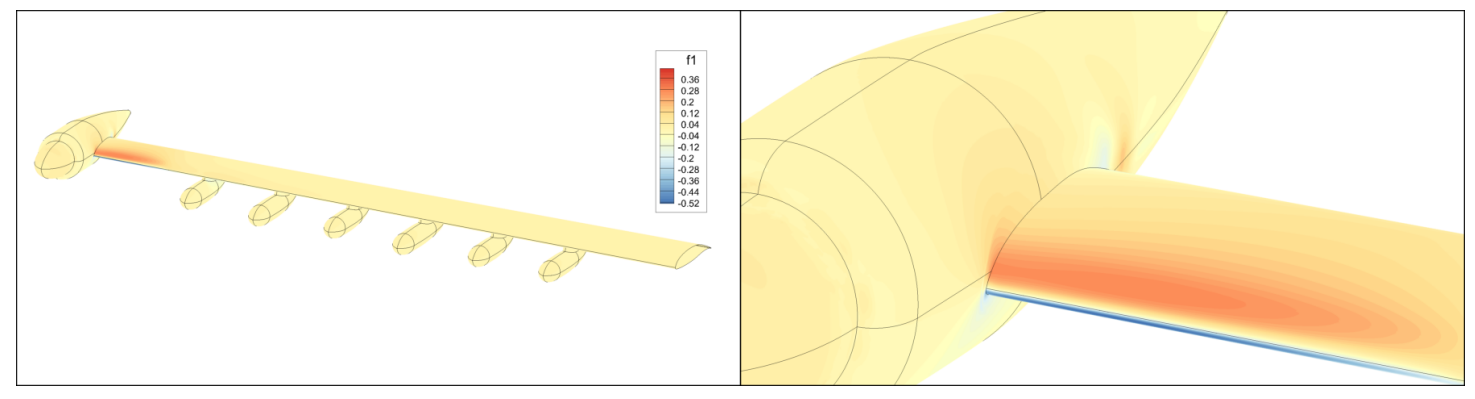

(a) Entire wing.

(b) Wing-nacelle junction.

Fig. 18 Difference induced due to propeller rotation at cruise RPM. (Pressure coefficients with the propellers operating - Pressure coefficients with stationary propellers).

shown in Figure 18. The propeller influences the aerodynamic distribution primarily at the wing tip leading edge. There is reduced, but possibly significant, influence over the entire chord near the wing tip, including the aileron. Work is ongoing to investigate the forced oscillation influence of the propeller wash on the aileron response.

\section{Ground Testing}

A series of tests are planned to measure the structural dynamic characteristics of the different vehicles and components. Because the configurations will not have wind tunnel assessments of the aeroelastic stability, these ground tests are essential to benchmark and correct the computational models. The aeroelasticity team views understanding the structural dynamic modes of the new components to be essential for conducting safe flight testing. Preliminary tests are planned for partially-assembled configurations when the hardware is available. This takes advantage of lead time for FEM correlation and subsequent analyses before final aircraft assembly, final ground vibration tests (GVTs) and flight testing. Component ground vibration tests are planned for newly fabricated components such as the propulsion systems and Mod III wing box. To date, a preliminary GVT of the Mod II configuration and isolated prop/hub GVTs have been conducted. These efforts and results are described below. In the upcoming months of 2018, a GVT of a simulated propeller/hub assembly with backup structure is planned. This effort will assess the influences of the vibration isolation system and motor mount structure. Also in the current year, we will conduct a full GVT of the final Mod II flight vehicle 


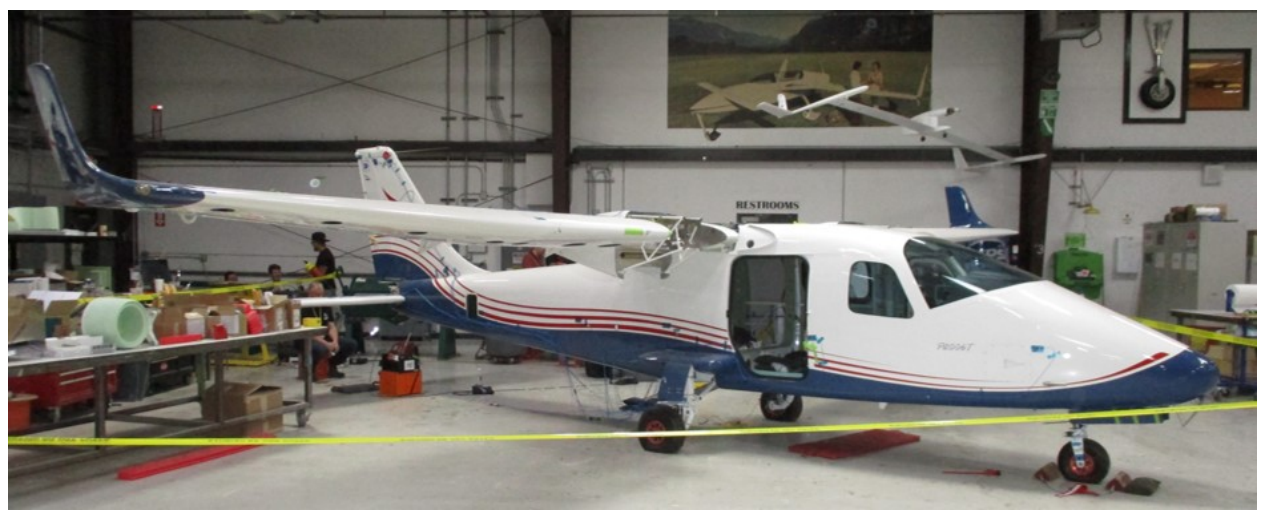

Fig. 19 GVT setup for "mini-GVT" of Mod II configuration.

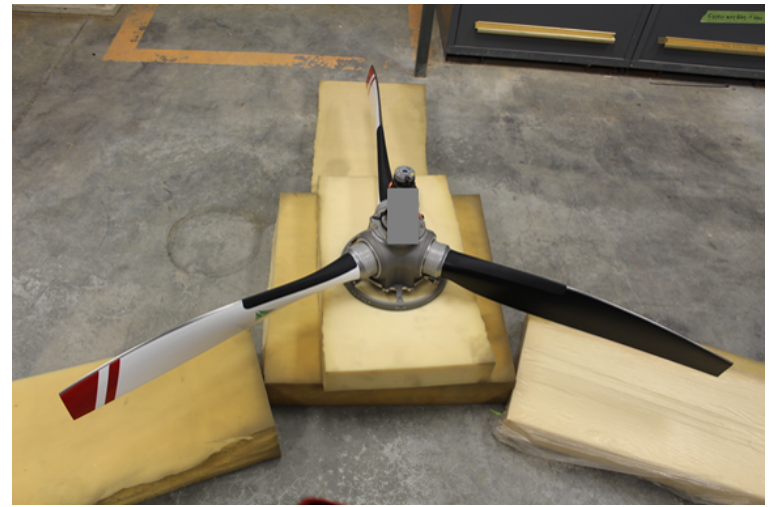

Fig. 20 GVT setup for measuring mode shapes and frequencies of the three-bladed prop and hub assembly.

and perform stiffness testing and ground vibration testing of the Mod III wingbox.

\begin{abstract}
A. Mod II mini-GVT
A "mini-GVT" was performed on the Mod II configuration in May 2017. The objective was to obtain preliminary frequencies and mode shapes to use in evaluating vehicle and whirl flutter. The aircraft was tested without the motors or prop, and with the landing gear and control surfaces constrained, as shown in Figure 19. This test was accommodated in the Mod II vehicle hardware modification plan, so the measurements could not be extensive. The primary area of focus was on the aft portion of the fuselage and the empennage. The most significant technical information to come from this testing was measurement of the stiffness associated with stabilator rotational freedom. This value was implemented in both the Mod II and Mod III revised models, showing significant influence of the flutter results, as previously discussed.
\end{abstract}

\title{
B. Prop/Hub structural dynamics testing
}

A preliminary structural dynamic test of a cruise propeller and hub was performed in August 2017. The objective of this "ping" test was to provide a preliminary assessment of the accuracy of the propeller blade structural modeling and to develop expertise in conducting testing on the propeller system. This testing feeds in to whirl flutter analysis that will not be further discussed in the current paper. The testing was conducted with the three-bladed propeller and hub supported on foam, shown in Figure 20, using hammer taps as the excitation source.

From an experimental point of view, the datasets were noisy, attributed to the teardrop accelerometers being mounted with wax. Blade modes were not measured during this preliminary testing, but the shapes were visually observed to be similar in shape to the analytical mode shapes. There were more modes present in the experiment than present in the single blade analysis. Analyses performed subsequent to this testing showed that these additional modes are attributable to multiple-blade symmetries. That is, for example, there is a blade bending mode where all blades bending 
simultaneously in the same direction. There are two other modes, identical in frequency, where two blades bend in the same direction, but the third blade bends in the opposite direction.

A ground vibration test of the cruise propeller and hub was subsequently performed in February 2018. The objectives of this test were to provide mode shapes and frequencies for updating the structural dynamic model of the rotating system that serves as the basis for whirl flutter assessments for both Mod II and Mod III vehicles. In this test, the hub was again set on foam to try and simulate a free-free boundary condition. Using the information from the ping test, the test matrix was expanded to include measurements with several propeller blade root rotational angles. Two types of accelerometers were used and more firmly mounted with hot glue. Teardrop accelerometers minimized the instrumentation weight, but tri-axial accelerometers, which are significantly heavier, improved the signal to noise ratio and provided much more mode shape definition.

Some lessons learned from this testing relative to the structural modeling include:

- The instrumentation weight is significant for this hardware; the computational model used in comparison to the experimental data should include the accelerometer masses.

- The single-blade mode shapes and frequencies are insufficient for comparison with the combined blade-hub system; the computational model should include all three blades and the hub.

- The rotated position of the blades (blade root angle relative to the hub) is significant.

\section{Status summary and plans}

The SCEPTOR project has progressed from a blank sheet of paper in 2015 to building and testing hardware. We hope to soon be flight testing the Mod II configuration. This paper has presented some of the current aeroelastic flutter predictions for the Mod II and Mod III configurations of the X-57. How well do these results reflect vehicle behavior? We won't know until after this paper is published and ground and flight data obtained. This paper serves as a results benchmark and a status report. We know that the tools that we are using to perform analyses are primarily low fidelity, but cost effective with the given resources and schedules. We investigate the issues that appear to be the most critical, improving our predictions using ground test information as it becomes available. In all predictions, we try to be conservative. Where the research objectives clash with the aeroelastic predictions, we sharpen our analyses or recommend design modifications or additional ground testing. There are three categories of problems that concern the SCEPTOR aeroelasticity team.

1. Problems that are standardly modeled and well-understood. At this point in time, we think that our baseline flutter analyses capture the aspects that we considered as likely problem areas at the project outset. Coupling of the first torsion mode with bending modes to produce classical flutter. Divergence of the long thin wing with a large tip mass. Coupling of the first bending mode with the rigid body degrees of freedom. Interference of the first bending mode with the pilot controls. These issues have been studied, designed for, and hopefully resolved.

2. Problems unique to this vehicle that we foresee at different points in the design, fabrication and ground testing. There are several of these issues, primary among them, whirl flutter. Closely related, is the influence of the propeller wash on the outboard section of the wing. For this type of issue, we have to figure out how much of an issue it is, and, if necessary, how to compensate or avoid it.

3. Something we never saw coming.

Our job within the SCEPTOR project is to try to limit the impact of aeroelastic restrictions on the performance research, vehicle development and flight testing, while producing a vehicle with sufficient safety margins. We continue to study and analyze, trying to understand items in category 2 and eliminate items in category 3.

The short term plans for the aeroelasticity team include completing the CFD-based flutter analyses of the Mod III cruise configuration. The cruise configuration will also continue to be the focus of the improvement in the correction factor scheme. The influence of the propeller operating will be assessed through linear flutter and forced response analyses. The linear flutter analyses for the Mod II and Mod III vehicles will be expanded to include input uncertainty propagation. The uncertain input variables will include mass locations, mass amounts, actuator stiffness values and modal frequency changes. 
Whirl flutter and interactions of rotating components with the other vehicle systems and structures are concerns of the aeroealsticity team. Some of the ground vibration testing has been reported on, but much remains to be done. Among the plans for the next several months are several ground tests relevant to gaining more confidence in the whirl flutter results and forced excitation effects.

The extended plans involve moving away from analyses of the cruise configuration and focusing on the take-off and landing configurations. Issues that have been resolved for the cruise configuration by analytically modeling the flap as stowed will almost certainly bear studying for the configurations where the flap is not stowed.

There is an array of ground testing planned for each configuration. Conducting these tests and comparing the results with analyses is a significant portion of the work to be done prior to flight test. Finally, our plans include preparing for flight testing where we will monitor the vehicles responses in real time and study the data post-flight to avoid making aeroelasticity front and center for the project.

\section{References}

[1] M.Moore, Goodrich, K., Viken, J., Smith, J., Fredericks, W., Trani, T., Barraclough, J., German, B., and Patterson, M., "High Speed Mobility through On-Demand Aviation," AIAA Paper 2013-4373, 2013. Aviation Technology, Integration and Operations Conference, Los Angeles, CA, August 2013.

[2] Moore, J. B., and Cutright, S., "Structural Design Exploration of an Electric Powered Multi-Propulsor Wing Configuration," AIAA Paper 2017-0203, 2017. AIAA SciTech Forum, 58th AIAA/ASCE/AHS/Structures, Structural Dynamics and Materials Conference, January 9-13, 2017, Grapevine, Texas.

[3] Bergqvist, P., "Tecnam P2006T: Tecnam's entry-level twin has the opportunity to move well beyond the flight school market," Flying, 2011. "https://www.flyingmag.com/aircraft/pistons/tecnam-p2006t".

[4] Hoover, C. B., and Shen, J., "Whirl Flutter Analysis of a Free-Flying Electric Driven Propeller Aicraft," AIAA paper, June 2018. To be presented at the AIAA Aviation Forum.

[5] https://www.zonatech.com/zaero.html, ZONA Technology, Inc., Scottsdale, AZ, 1985-2018.

[6] http://www.mscsoftware.com/product/msc-nastran, MSC Software Corporation, Newport Beach, California, USA, 2017.

[7] http://www.mathworks.com/products/matlab/, The MathWorks, Inc. Natick, Massachusetts, USA, 1994-2018.

[8] Massey, S. J., Wieseman, C. D., Stanford, B. K., and Heeg, J., "Aeroelastic analysis of a Distributed Electric Propulsion Wing," AIAA Paper 2017-0413, 2017. AIAA SciTech Forum, 58th AIAA/ASCE/AHS/Structures, Structural Dynamics and Materials Conference, January 9-13, 2017, Grapevine, Texas.

[9] Pointwise V17.3R3 User Manual, Pointwise, Inc., Fort Worth, Texas, 2015.

[10] Anon., "DPW6 Gridding Guidelines,” 2015. "URL:http://aiaa-dpw. larc.nasa.gov/Workshop6/grids.html".

[11] Biedron, R. T., Carlson, J.-R., Derlaga, J. M., Gnoffo, P. A., Hammond, D. P., Jones, W. T., Kleb, B., Lee-Rausch, E. M., Nielsen, E. J., Park, M. A., Rumsey, C. L., Thgomas, J. L., and Wood, W. A., FUN3D Manual: 13.3, 10.6.0 Manual, NASA LaRC, Hampton, VA, feb 2018. http: //fun3d. larc . nasa. gov.

[12] Spalart, P. R., and Allmaras, S. R., “A One-Equation Turbulence Model for Aerodynamic Flows,” , 1994. La Recherche Aerospatiale, No. 1, pp. 5-21.

[13] Spalart, P. R., "Strategies for Turbulence Modelling and Simulations," International Journal of Heat and Fluid Flow, Vol. 21, No. 3, 2000, pp. 252-263.

[14] Shur, M. L., Strelets, M. K., Travin, A. K., and Spalart, P. R., "Turbulence Modeling in Rotating and Curved Channels: Assessing the Spalart-Shur Correction,” AIAA Journal, Vol. 38, No. 5, 2000, pp. 784-792.

[15] Roe, P. L., "Approximate Riemann Solvers, Parameter Vectors, and Difference Schemes," Journal of Computational Physics, Vol. 43, 1981, pp. 357-372.

[16] Nyukhtikov, M., Smelova, N., Mitchell, B. E., and Holmes, D. G., "Optimized Dual-Time Stepping Technique For Time-Accurate Navier-Stokes Calculations," Proceedings of the 10th International Symposium on Unsteady Aerodynamics, Aeroacoustics and Aeroelasticity of Turbomachines, 2003. 
[17] Vatsa, V. N., Carpenter, M. H., and Lockard, D. P., "Re-evaluation of an Optimized Second Order Backward Difference (BDF2OPT) Scheme for Unsteady Flow Applications,” AIAA Paper 2010-0122, Jan. 2010.

[18] Thomas, P. D., and Lombard, C. K., “Geometrical Conservation Law and Its Application,” AIAA Journal, Vol. 17, No. 10, 1978, pp. 1030-1037.

[19] Biedron, R. T., and Thomas, J. L., "Recent Enhancements to the FUN3D Flow Solver for Moving-Mesh Applications," AIAA Paper 2009-1360, Jan. 2009.

[20] Cunningham, H. J., Batina, J. T., and Bennett, R. M., "Modern wing flutter analysis by computational fluid dynamics methods," Journal of Aircraft, Vol. 25, No. 10, 1988, pp. 962-968. doi:10.2514/3.45686.

[21] Rendall, T., and Allen, C., "Efficient Mesh Motion Using Radial Basis Functions with Data Reduction Algorithms," Journal of Computational Physics, Vol. 228, 2009, pp. 6231-6249. doi:10.1016/j.jcp.2009.05.013.

[22] Drela, M., and Youngren, H., XROTOR download page, http://web.mit.edu/drela/Public/web/xrotor, 2018. 